\title{
Quardicuspid Aortic Valve as Risk Factor for Infective Endocarditis
}

\author{
Yahya Islamoglu ${ }^{1}$, Cansu Tokat ${ }^{2}$, Sumeyra Uysal ${ }^{3}$ \\ ${ }^{1}$ Dicle University Faculty of Medicine, Department of Cardiology, Diyarbakir, Turkey \\ ${ }^{2}$ Uludag University Faculty of Medicine, Bursa, Turkey \\ ${ }^{3}$ Düzce University Faculty of Medicine, Düzce, Turkey
}

\begin{abstract}
Quardicuspid aortic valve, an infective endocarditis's risk factor, has the property inborn aortic valve disease but disease development not up to enough understood. Infective endocarditis is together with 1, 4\% of the cases. Aortic regurgitation may be seen together and transesophageal echocardiography is important for these patients because of diagnosis. Our case, a thirty nine years old woman, was asymptomatic quardicuspid aortic valve with aort valve regurgitation and we diagnosed via transesophageal echocardiography. We recommended antibiotic prophylaxis and annual follow to the patient. J Microbiol Infect Dis 2018; 8(1):30-32
\end{abstract}

Keywords: Infective endocarditis, quadricuspid aortic valve, aortic regurgitation

\section{INTRODUCTION}

Quardicuspid aortic valve (QAV), average incidence of $0,0003 \%$ in autopsy examinations, is an infrequent congenital heart disease determined by the Balinton in 1862 [1]. Also QAVs are increasingly reported by advance imaging diagnostic techniques such as transthoracic echocardiography (TTE), transesophageal echocardiography (TEE) [2]. But the mechanisms of QAV development are not known precisely. According to the scientists, unnatural septation occurs in endocardial cushions because of formed inflammation [3]. Thus, in the truncus arteriosus whom is formed unnatural mesenchymal changes, is happened quardicuspid pattern formation, and/or in aorticopulmonary septum is happen unnatural fusion for its [4]. The aortic regurgitation (AR) is occurred with QAV and is the most common complication. In our case report, there is a patient that asymptomatic QAV with AR diagnosed via TEE.

\section{CASE REPORT}

Our hospital admitted a thirty nine years old woman patient for evaluation of diastolic heart murmurs. The patient had no previous history of cardiovascular disease and any symptom. Upon presentation, vitals were as follows: oral temperature of $37,5^{\circ} \mathrm{C}$, blood pressure of $140 / 70 \mathrm{mmHg}$, heart rate of 90 beats/min, and respiratory rate 18 breaths/min, with an oxygen saturation of $98 \%$ on room air. There was no any abnormality on physical examination apart from $4 / 6$ diastolic murmur along the left sternal border. An electrocardiogram showed left ventricular high voltage in addition to mild upsloping ST segment depression in V5 and V6 leads. The chest radiogram showed normal findings.

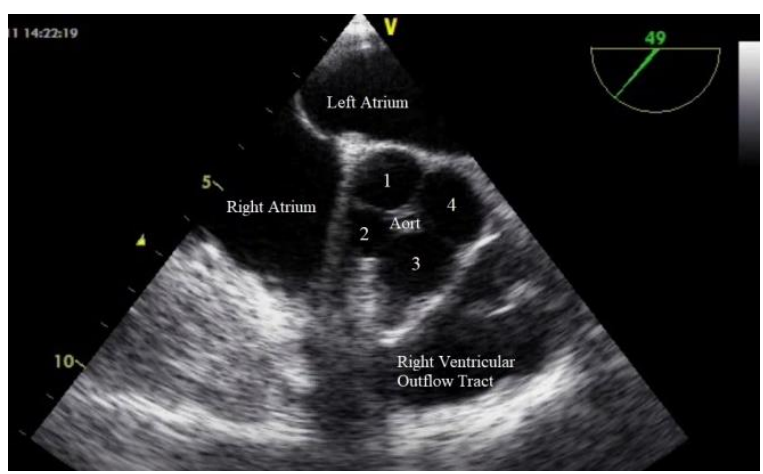

Figure 1. See Figure 5.

The patient declined to perform TTE. It demonstrated mildly left ventricle dilatation and normal ejection fraction, moderate $A R$ related probable QAV. No other pathology was noted. A TEE, performed to better define her valvular anatomy, showed a QAV (Figure 1, 2) with 
preserved valve opening motion pattern and moderate AR (Figure 3). Antibiotic prophylaxis for infective endocarditis (IE) and annual follow for AR were recommended to the patient.

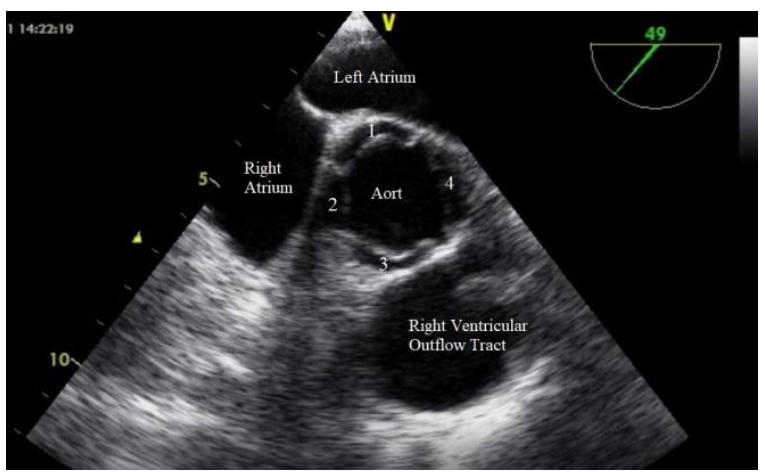

Figure 2. See Figure 5.

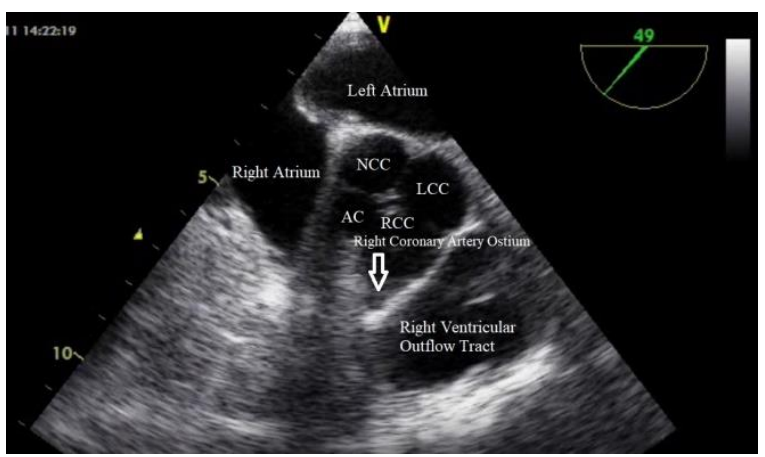

Figure 3. Transesophageal echocardiography showing 2-3 (moderate) degree aortic valve regurgitation $(A R)$.

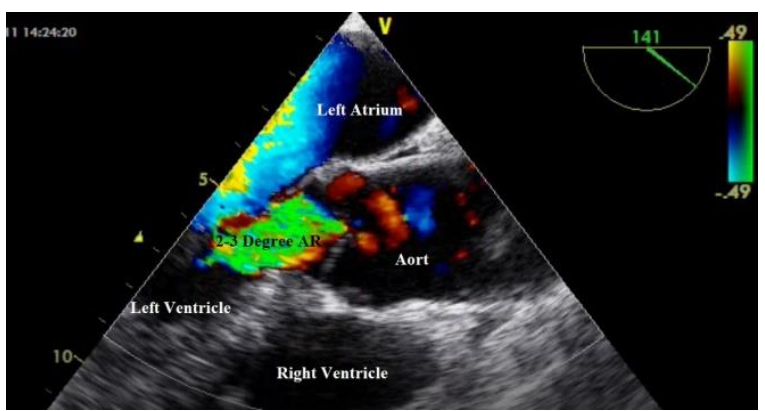

Figure 4. See Figure 5.

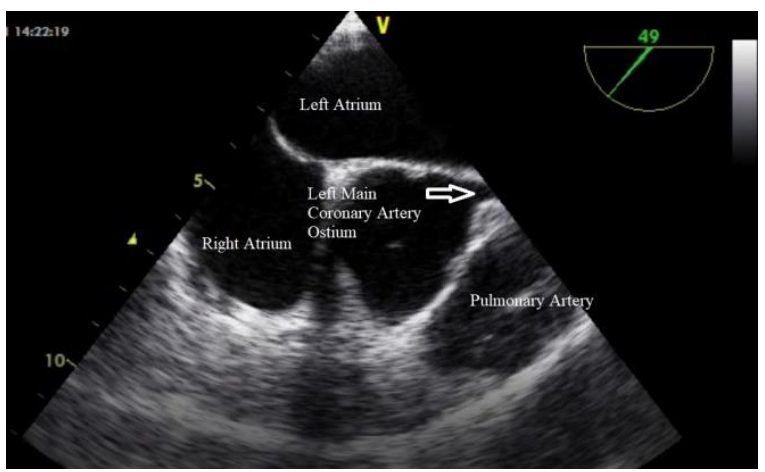

Figure 5. (Figures 1, 2, 4 and 5) Transesophageal echocardiography showing an aortic valve with four cusps, and left and right coronary artery ostium [left coronary cusp (4, LCC), right coronary cusp (3, RCC), non-coronary cusp (1, NCC) and accessory cusp (2, $A C)]$.

\section{DISCUSSION}

The QAV, rarely seen as inborn aortic valvular disease, isn't known exactly how it happens. Unnatural septation of embryological arterial trunk was the one of the most important theory. According to this theory, 4th leaflet formation occurs early phase of truncal septation.

In this early phase occurs unnatural aorticopulmonary septation or unusual cusp progression [5,6]. As a result of this, the ventriculoaortic junction becomes deformed geometric shape according to normally.

If a person has QAV, that person should also be carefully evaluated for other congenital abnormalities. QAV have been reported in association with nonobstructive cardiomyopathy, vavular stenosis (such as pulmonary, aortic or subaortic), ventricular septal defect and coronary artery anomalies (it was not there in our case, Figure 4, 5) [7, 8]. Nowadays TEE play an important role in notice QAV. In patients with QAV, continual follow-up is advised, in view of the eventual requirement of aortic valve surgery. Also IE is a potential complication [9]. In our case, no cardiovascular and other system abnormalities associated with QAV were there.

Hurwitz et al. [7] and Nakamura et al. [10] performed different two classification and we are classifying the patient with QAV according to anatomies since that time. Our patient had "Type B QAV" according to classification of Hurwitz et al. (3 equal large cusps and one smaller cusp), and "Type II QAV" according to classification of Nakamura et al.

Extra small cusps or similar other structures can be a risk factor for IE [11]. The unequal cusps the risk of $I E$ is higher owing to absence of symmetry, and stream disturbance. In the dissymmetry aort leaflets, because of unequal dispersion of stress and uncompleted juxtaposition during diastole occurs gradual AR and progressive disruption in course of time, and thus increasing the risk for IE [2]. Therefore, earlier identification of QAV with AR has vital in terms of the high risk of IE [12]. But in the patients, discrepancy still remains regarding 
prophylaxis of IE. While part of the authors advised for absolutely antibiotic prophylaxis [13], others proposed in patients with AR and these patients had an extra small cusp, all of them were not equal size [11]. In our case had moderate AR but asymptomatic and we recommended annual following with antibiotic prophylaxis of endocarditis to the patient.

In conclusion, QAV is an infrequently congenital heart disease noted in adult life, incidentally with TTE. TEE's significance is increasing day by day even more for clinicians. Also infection should keep in mind for such cases.

\section{ACKNOWLEDGMENTS}

Conflict of interest: The authors declare no personal or financial conflict of interest.

Financial Disclosure: No financial support was received

\section{REFERENCES}

1. Cheema MA. Quardicuspid aortic valve as cause of congestive cardiac failure: case history. Pak Heart J 1990; 23(1): 14-15.

2. Malviya A, Jha PK, Ashwin, Mishra J, Srivastava P, Mishra A. Quardicuspid aortic valve: a case report and literature review. Egypt Heart J. 2016; 68(4):271-275.

3. Savino K, Quintavalle E, Ambrosio G. Quardicuspid aortic valve: a case report and review of the literature. J Cardiovasc Echography 2015; 25: 72 76.

4. Attaran RR, Habibzadeh MR, Baweja G, Slepian MJ. Quardicuspid aortic valve with ascending aortic aneurysm: report a case and discussion of embryological mechanisms. Cardiovasc Pathol 2009; 18(1): 49-52.

5. Brouwer MHJ, de Graaf JJ, Ebels T. Congenital quardicuspid aortic valve. Int J Cardiol 1993; 38: 196-8.

6. Davia JE, Fenoglio JJ, De Castro CM, McAllister HA, Cheitlin MD. Quardicuspid semilunar valves. Chest 1977; 72: 608-610.

7. Hurwitz LE, Roberts WC. Quardicuspid semilunar valves. Am J Cardiol 1973; 31(5): 623-626.

8. Timperley J, Milner R, Marshall AJ, Gilbert TJ. Quardicuspid aortic valve. Clin Cardiol 2002; 25 (12): 548-552.

9. Bauer F, Litzler PY, Tabley A, Cribier A, Bessou JP. Endocarditis complicating a congenital quardicuspid aortic valve. Eur $\mathrm{J}$ Echocardiogr 2008; 9(3): 386-387.
10. Nakamura Y, Taniguchi I, Saiki M, Morimoto K, Yamaga T. Quardicuspid aortic valve associated with aortic stenosis and regurgitation. Jpn J Thorac Cardiovasc Surg 2001; 49: 714-716.

11. Takeda N, Ohtaki E, Kasegawa H, Tobaru T, Sumiyoshi T. Infective endocarditis associated with quardicuspid aortic valve. Jpn Heart J 2003; 44(3): 441-445.

12. Bilge AK, Bugra Z, Tayyareci Y, Ruzgar O, Umman S, Meric M. An uncommon coincidence of congenital quardicuspid aortic valve accompanied by hypertrophic obstructive cardiomyopathy. Anadolu Kardiyol Derg 2007; 7(4):E7-E8.

13. Kawanishi $Y$, Tanaka H, Nakagiri K, Yamashita T, Okada K, Okita Y. Congenital quardicuspid aortic valve associated with severe regurgitation. Asian Cardiovasc Thorac Ann 2008; 16(5):e40-e41. 